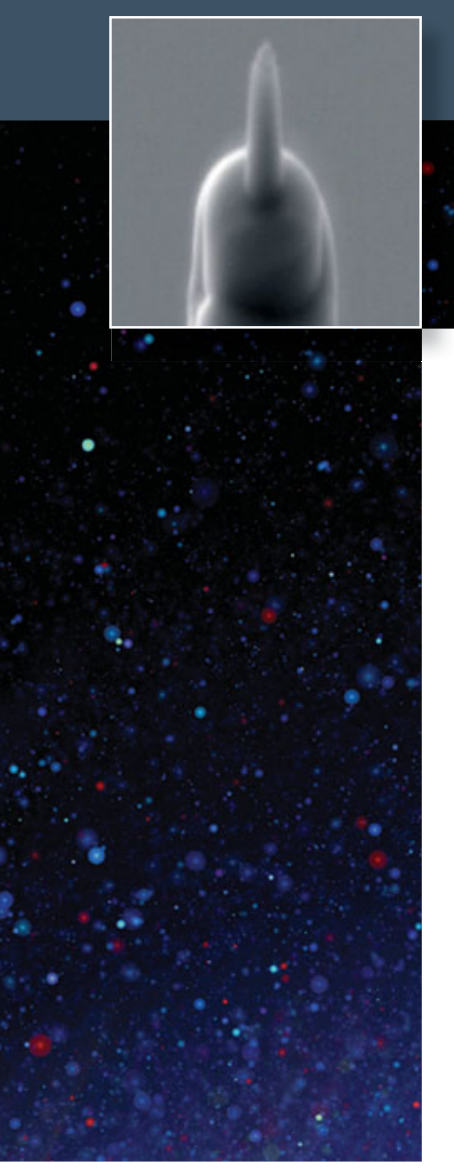

\title{
Atom probe tomography-A cornerstone in materials characterization
}

\author{
Yaron Amouyal and Guido Schmitz, Guest Editors
}

\begin{abstract}
Frequently, fundamental scientific and technological issues are related to the chemical structure of a material at the nanometer or even atomistic length scales. This includes, but is not limited to, internal interfaces of complex topology as they appear, for example, in current energy-harvesting applications or advanced microelectronics. Scientific understanding of the underlying physics and chemistry requires advanced characterization tools that provide critical three-dimensional information at the subnanometer length scale. Atom probe tomography (APT) meets such requirements. Today, with remarkable progress in instrumentation and sample preparation, APT has become a very versatile tool to address fundamental questions of materials science. In this issue of MRS Bulletin, the APT technique is introduced, with a particular focus on recent developments and the broadening range of studied material classes and applications.
\end{abstract}

\section{Basics and principles}

Atom probe tomography (APT) is based on controlled field ionization and evaporation of individual atoms from the surface of needle-like samples that are sharpened to an apex radius of less than $100 \mathrm{~nm}$. The evaporated ions are accelerated toward a position-sensitive multichannel plate detector that records the $\left(X_{\mathrm{DET}}, Y_{\mathrm{DET}}\right)$-impact position and the time of flight, $t$, atom-by-atom. Subsequently, the original $(x, y$, and $z)$ locations of the atoms inside the specimen are derived through quantitative evaluation of the detection sequence and the detected positions. This reconstruction procedure performs surprisingly well, reaching a few Angstroms accuracy in suitable cases.

The chemical identity of the ions is determined by their time of flight. For this, the field evaporation has to be triggered either by superposed additional electrical field pulses or by short laser pulses. The characteristic mass-to-charge-state ratio, $m / n$, is then determined by:

$$
m / n=2 q V\left(\frac{t}{d}\right)^{2},
$$

with $q, V, t$, and $d$ denoting the elementary charge, applied voltage, time of flight, and flight distance, respectively. This is a sufficient approximation derived from conservation of energy under the assumption of instantaneous acceleration close to the tip's surface. A complete compositional analysis is obtained by counting the number of ions having a given $\mathrm{m} / \mathrm{n}$ ratio. This yields a spectrum, in which even different isotopes of the elements are usually resolved, appearing as a series of peaks with intensities proportional to the natural isotope abundance. ${ }^{1-6}$

The principle of this particular kind of analysis has been known for decades. What has recently made APT so attractive are the decisive technical improvements that have turned modern APT instruments into versatile tools for practical materials analysis. Enabled by rapid modern-detection electronics, the aperture angle (field of view) has been enhanced, and is currently about 50 times larger than what conventional atom probes have been able to display. In an advanced APT design, a local extraction electrode is placed about $30 \mu \mathrm{m}$ in front of the tip, ${ }^{1-6}$ such that the high voltage necessary to achieve the required evaporation field is significantly reduced. In turn, thicker samples can be evaporated at a given voltage.

Perhaps the most important progress in this field in the last decade has been the establishment of laser-assisted evaporation modes. Instead of supplying negative voltage pulses to the extraction electrode, flashing ultrashort light to the tip apex triggers the evaporation, thus providing the necessary activation for the final evaporation step. As its major advantage, the laser pulsing opens the analysis to nonconducting materials. Further welcome side effects are the ability of producing higher evaporation frequencies, up to $1 \mathrm{MHz}$, 
and a higher mass resolution, as the ion acceleration is no longer disturbed by the voltage pulses. ${ }^{5,6}$

In a recent (2009) design of the laser-beam line, an in-vacuum optics system focuses a smaller $(0.5$ - $\mu$ m diameter $)$, diffraction-limited ultraviolet (UV, wavelength $\lambda=355 \mathrm{~nm}$ ) laser spot on the tip apex. This reduces the heat-affected zone and thus accelerates the cooling with further improved mass resolution. ${ }^{7,8}$ In modern instruments, a mass-resolving power of $m / \Delta m=800-1000$ can be typically achieved, which may even be increased to 1200 using an optimized UV laser system. ${ }^{9-12}$ Here, $m$ denotes the ion's mass determined at the peak center, and $\Delta m$ is the peak width (full width at half maximum). Collection rates amount to $10^{8}$ atoms $\mathrm{h}^{-1}$. With the enhanced field of view, the analysis volume can reach $200 \times 200 \times 1000 \mathrm{~nm}^{3}$, although this benchmark depends largely on the studied material and the tip geometry.

The latest technical advancement, which may become decisive in the study of short-range order or in the analysis of very small clusters, involves improvement of the sensitive area ratio of the multichannel plates, which increases the detector efficiency from about $50 \%$ to $75 \%$ of the evaporated species. The latter could even be further improved by placing an electron mirror in front of the detector surface, although this bears a risk of degradation in spatial resolution. ${ }^{57}$

\section{Applications in materials science}

At present, various areas of materials science benefit from utilizing APT, including metallurgy, microelectronics, polymer science, biogenic materials, and advanced ceramics. The following is a partial list of scientific and technological questions that can be addressed with the help of APT, usually in combination with the different modes of transmission electron microscopy (TEM).

- Can we trace the earliest stages of microstructure evolution?

Fine control of the nanostructure evolution of alloys, including clustering, nucleation, precipitate growth, and coarsening, is particularly critical in the development of advanced alloys for high-temperature applications. ${ }^{13-23}$ In this issue of MRS Bulletin, Seidman et al. introduce typical physical metallurgy issues in which APT plays a decisive role.

- What is the role of minority elements?

This question concerns such important areas in materials science as the distribution of dopants at the vicinity of heterojunctions, interfacial energetics in metallic and ceramic systems, and impurities around the roots of microcracks affecting mechanical behavior and other properties. High sensitivity for impurity elements with concentrations as low as 50 at. ppm is one of the capabilities of modern APT, originating from a high signal-tonoise ratio and good mass-resolving power. For example, the role of Hf (0.05 at.\%) in Ni-based alloys has been demonstrated using APT. ${ }^{24,25}$
- Can we detect and observe nanometer-sized core-shell structures?

For this purpose, three-dimensional (3D) spatial resolution combined with the detection and quantification of elements that are distributed at nanometer length scales is required. Elemental mapping in core-shell nanostructures has been performed using APT for different geometries (e.g., mapping of quantum wells in $\mathrm{GaN} / \mathrm{InGaN}$ core-shell nanowires $[\mathrm{NWs}])^{26}$ or quantitative $3 \mathrm{D}$ analysis of the distribution of germanium atoms within Ge-dome islands buried in a silicon matrix, see Figure 1. ${ }^{27}$ Remarkably, core-shell nanostructures were not considered to be a common phenomenon in metallic systems, mainly because they could not be easily detected employing conventional electron microscopy. However, thanks to the new capabilities of the APT technique, they have been observed and chemically analyzed. Core-shell nanostructures have been observed by Monachon et al., ${ }^{28}$ attributed to the excellent spatial resolution that can be obtained for complex topologies. Krug et al. have reported additional APT analysis of core-shell precipitates for aluminum-scandium-based systems. ${ }^{29,30}$

- How to quantify the size distribution of precipitates in metallic matrices?

Precipitate analysis, including size and edge-to-edge distribution functions, are common applications of APT. ${ }^{20}$ Prominently, the distributions of nanometer-sized $\gamma^{\prime}$-precipitates in a $\gamma$-matrix in Ni-based alloys have been obtained using APT. ${ }^{31}$ Quantitatively, new APT devices of their different generations enable us to sample volumes containing nanometer-sized precipitates with number density values ranging between $10^{21}$ and $10^{25} \mathrm{~m}^{-3}$, thus yielding precipitate size distribution.

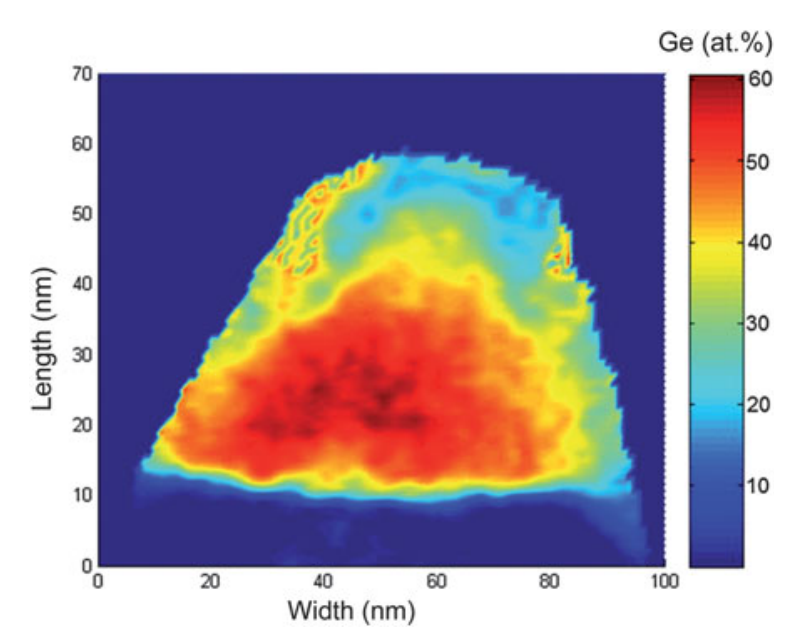

Figure 1. Two-dimensional projected atom probe tomography (APT) reconstruction of the Ge distribution measured around the core of a Ge island in a Si matrix in a single APT volume. Reproduced with permission from Reference 27. () 2012 AIP Publishing LLC. 
In this context, it is noteworthy that an emerging application of APT is the analysis of precipitate-size distribution, number density, and morphology, as well as solute concentrations in the matrix of two-phase thermoelectric (TE) materials. ${ }^{32-37}$ Such an analysis is essential to elucidate the TE performance in correlation to the microstructure. The heat-to-electricity conversion efficiency is enhanced by reducing the thermal conductivity of a material without degrading the electrical conductivity and thermopower. ${ }^{38}$ This can be achieved by introducing precipitates with high number densities that serve as phonon-scattering centers, thereby reducing the mean free paths of the latter. ${ }^{39-41}$

Biswas et al., ${ }^{32}$ who investigated PbTe-based compounds, reported a striking example for the application of APT to TE materials. They observed segregation of Na solute atoms to $\mathrm{PbTe} / \mathrm{SrTe}$ interphase boundaries and to dislocation cores. They suggested that $\mathrm{Na}$ atoms are confined to these defects at low temperatures; however, these are released into the matrix at elevated temperatures, giving rise to $p$-type conduction. This example demonstrates how functional properties may be explained using the microstructure and composition analysis acquired by APT.

- Can APT handle cases with extreme topologies?

APT can serve the microelectronics industry by enabling analysis of the spatial distribution of dopants in extremely low concentrations, across topologically complex interfaces. ${ }^{42,43}$ APT has become an essential tool for analyzing dopant distribution along the principal and radial axes of NWs for nanoelectronicsbased devices. ${ }^{44,45}$ For example, in efforts to replace gold with aluminum as catalysts for NW growth, Moutanabbir et al. mapped Al-impurity atoms in vapor-phase-grown silicon NWs using TEM and APT, see Figure 2. ${ }^{45}$ This exemplifies the power of combining TEM and APT techniques and has prime technological implications for synthesis of NW-based nanoelectronics devices. In this issue of MRS Bulletin, the article by Larson et al. focuses on the characterization of nanoscale electronic materials, including fin-shaped field-effect transistors (FinFETs), the microstructure of silicide contacts, NWs, and quantum dots.

- Can we collect and quantitatively analyze ionic or covalently bound complexes in systems that are chemically more complicated than metallic materials?

Investigations of macromolecular materials by APT is a rather young and still relatively new field. ${ }^{46-49}$ As a rule, complex mass spectra with a large number of molecular fractions are observed, but often, the majority of peaks can still be identified. Challenges in evaporation and chemical analysis of large ionic complexes have recently been overcome in the application of APT to biogenic materials.$^{50}$ For example, Gordon et al. have mapped bone and dentine organic tissues by analyzing chemical derivatives of apatite (the mineral constituents of these tissues) using APT. ${ }^{51}$ These studies are important for understanding the mechanical functioning of the human skeleton. Moreover, the extraordinarily large surface area of the apatite acts as an ion-exchanger for essential and toxic ions in the human body. Gordon et al. have demonstrated the application of laser-pulsed APT to chemically analyze organic complexes in tissues and map them, see Figure 3. ${ }^{51}$ They also introduced 3D chemical maps of organic fibers having 5-10 nm diameters in surrounding nanocrystalline magnetite $\left(\mathrm{Fe}_{3} \mathrm{O}_{4}\right)$ mineral in the tooth of the chiton Chaetopleura apiculate, which is a type of marine mollusk. ${ }^{52}$ Such novel applications are made possible by fine-tuning the laser power and reducing it to adequately low values appropriate for materials with low thermal conductivity. ${ }^{9}$

- Is APT applicable to conductive materials only? Application of APT to nonconducting materials such as advanced ceramics has been a challenge that was overcome employing the option of laser-pulsing. ${ }^{53,54}$ For example, Marquis and co-workers analyzed the chemical composition and

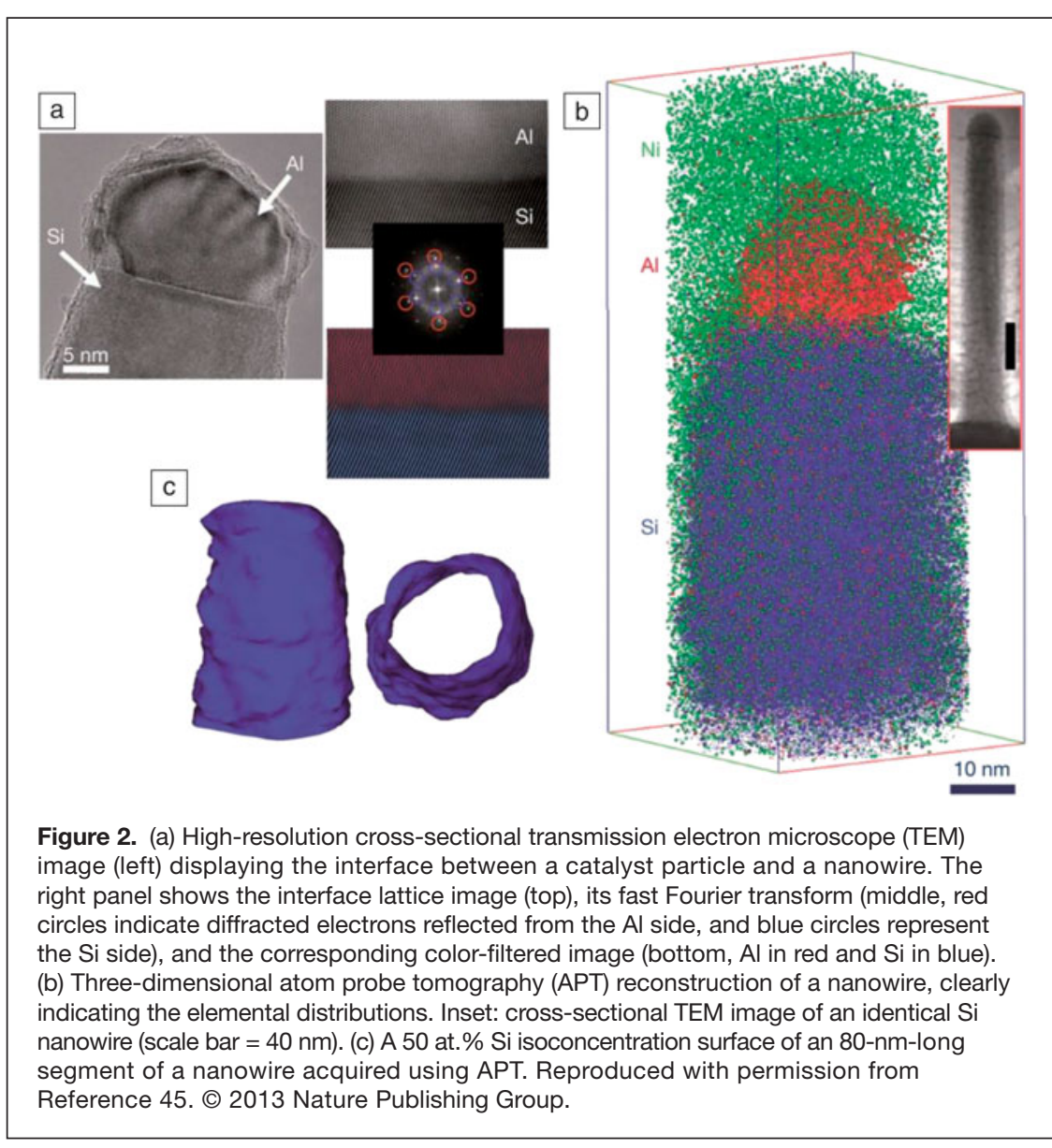




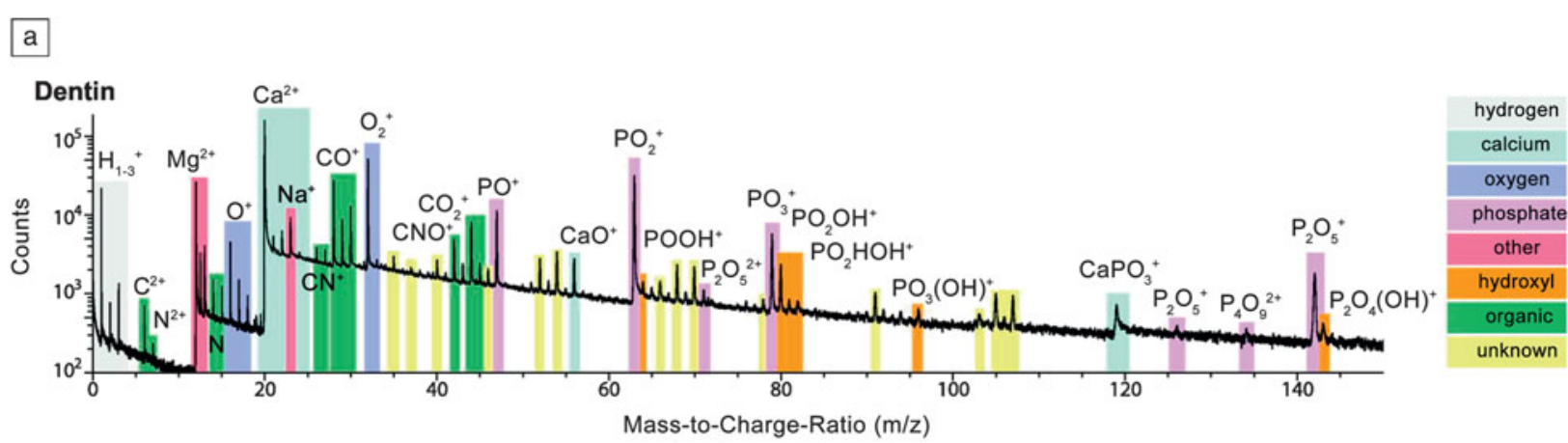

$\mathrm{b}$
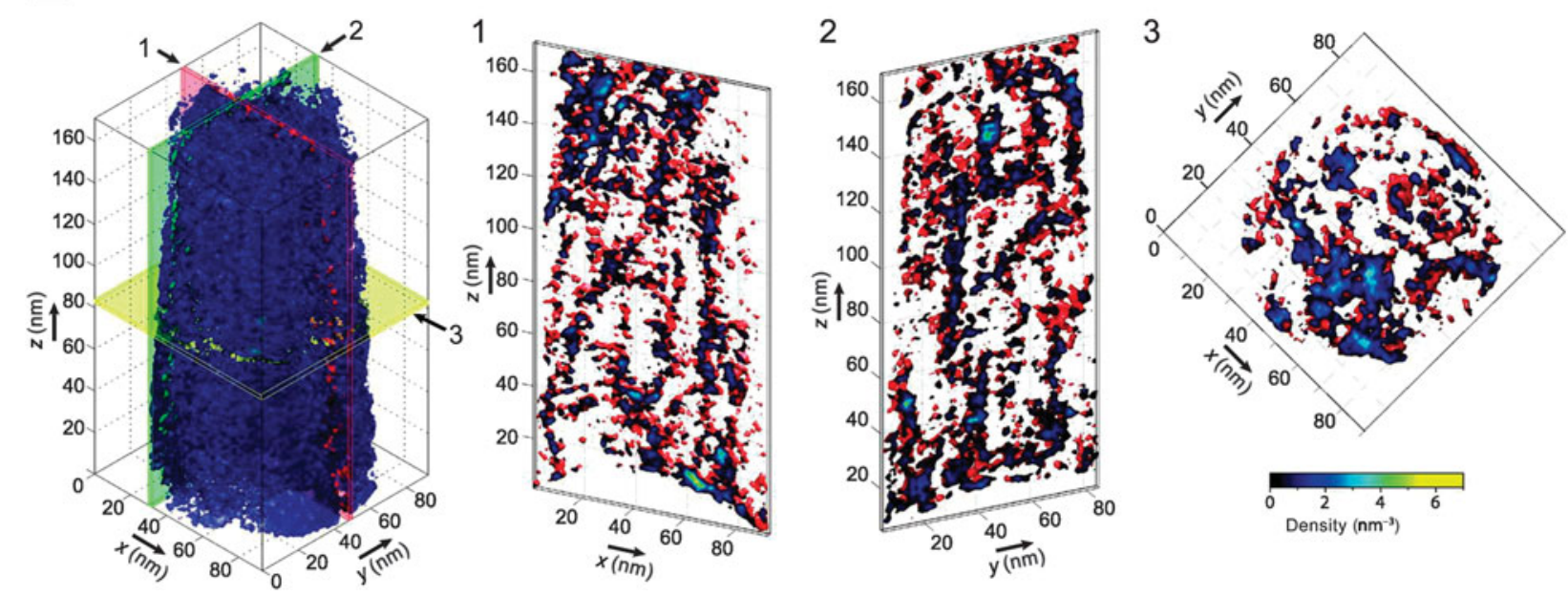

Figure 3. (a) lonic mass-to-charge-state spectrum of elephant tusk dentin indicating the hydrogen, calcium, oxygen, phosphate, other inorganic ions, and identified organic molecular fragments. Identification of the organic molecular fragments was made possible by application of low-energy laser pulses of $150 \mathrm{pJ}$ with a $200-\mathrm{kHz}$ repetition rate. (b) Three-dimensional (3D) rendering of the same samples showing (1) isoconcentration surface of the number density of organic fragment ions and the corresponding ([1] through [3]) slices across the principal axes. This yields complete 3D mapping of fibrous organic structures, which may correspond to individual collagen microfibrils and their assemblies. Reproduced with permission from Reference 51. (c) 2012 American Chemical Society.

microstructural features in alumina. ${ }^{54}$ They described APT analysis of carbon segregation at grain boundaries, which is barely achievable by analytical electron microscopy (see Figure 4). These findings help in understanding the effects of dopants on mechanical properties, such as fracture and wear, of advanced ceramics. Further insights from APT investigations of interfaces in ceramic films and layers are given in the review by Stiller et al. in this issue.

\section{Recent developments in APT analysis techniques}

The evolution of the APT technique is manifested by its ability to shift from traditional applications with crystalline metals to modern problems related to semiconducting and nonconductive solids, soft matter, and biogenic materials. The development of new data-acquisition technologies has enabled new operation modes, which has led to new applications. Among these technologies are fast-delay line detectors, which significantly extend collection angles and field-of-view, laser-assisted evaporation modes, including short-wavelength lasers in the UV regime and tunable laser energy that enable analyzing nonconductive materials, and a reflectron-mirror lens for improved mass-resolving power for high-voltage pulsing, as well as a local extraction electrode that offers advantages in sample handling and voltage requirements. ${ }^{6,55,56}$ Actual trends in the development of instrumentation are further discussed in the article by Moody et al. in this issue. However, more importantly, they place a special focus on "side" equipment such as the recently developed in situ gas reactions cells, which are now available as an integrated part of the instrument, and cryo-transfer systems, which are currently under development.

Once data are collected, post-analysis processing is required to achieve the desired 3D atomic maps from the raw data. Presently, trajectory aberrations in the case of heterogeneous samples with contrasting evaporation thresholds are the most serious challenge in deriving reliable volume reconstruction. Additionally, the advantage of collecting information on millions of atoms individually requires the development 

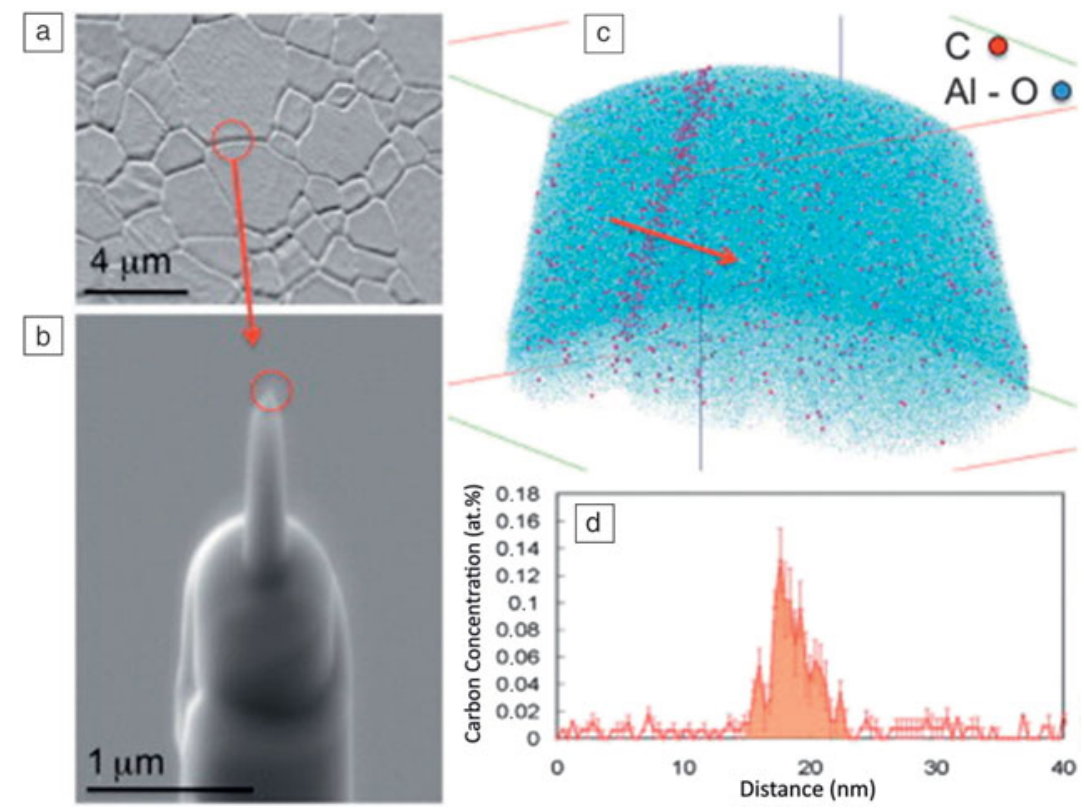

Figure 4. (a) Scanning electron microscope micrograph showing the etched surface of an alumina sample revealing grain-boundary surface grooves. (b) An atom probe tomography (APT) specimen lifted out from the alumina surface using dual-beam focused ion beam. The specimen contains a grain boundary near its apex. (c) The resulting threedimensional APT reconstructed volume showing carbon segregation at an alumina grain boundary, from which (d) a carbon concentration profile across the grain boundary (red arrow in [c]) is extracted. Reproduced with permission from Reference 54. (C) 2010 Elsevier.

project support and the German Federal Ministry for support in instrumental development (BMBF VIP 0370).

\section{References}

1. T.F. Kelly, D.J. Larson, Mater. Charact. 44 (1/2), 59 (2000).

2. T.F. Kelly, T.T. Gribb, J.D. Olson, R.L. Martens, J.D. Shepard, S.A. Wiener, T.C. Kunicki, R.M. Ulfig, D.R. Lenz, E.M. Strennen, E. Oltman, J.H. Bunton, D.R. Strait, Microsc. Microanal. 10 (3), 373 (2004)

3. M.K. Miller, Atom Probe Tomography (Kluwer Academic/ Plenum Publishers, New York, 2000).

4. D.N. Seidman, Annu. Rev. Mater. Res. 37, 127 (2007).

5. T.F. Kelly, M.K. Miller, Rev. Sci. Instrum. 78 (3) 031101 (2007).

6. D.N. Seidman, K. Stiller, MRS Bull. 34 (10), 717 (2009). 7. O. Moutanabbir, D. Isheim, D.N. Seidman, Y. Kawamura, K.M. Itoh, Appl. Phys. Lett. 98 (1), 013111 (2011).

8. M.D. Mulholland, D.N. Seidman, Microsc. Microanal. 17 (6), 950 (2011).

9. Y. Amouyal, D.N. Seidman, Microsc. Microanal. 18 (5), 971 (2012).

10. J. Bunton, J. Olson, D. Lenz, D.J. Larson, T.F. Kelly, Microsc. Microanal. 16 (Suppl. S2), 10 (2011).

11. Y.M. Chen, T. Ohkubo, K. Hono, Ultramicroscopy 111 (6), 562 (2011).

12. K. Hono, T. Ohkubo, Y.M. Chen, M. Kodzuka, K. Oh-ishi, H. Sepehri-Amin, F. Li, T. Kinno, S. Tomiya, Y. Kanitani, Ultramicroscopy 111 (6), 576 (2011).

13. C. Booth-Morrison, R.D. Noebe, D.N. Seidman, Acta Mater. 57 (3), 909 (2009).

14. C. Booth-Morrison, J. Weninger, C.K. Sudbrack, Z. Mao, R.D. Noebe, D.N. Seidman, Acta Mater. 56, 3422 (2008).

15. Z. Mao, C.K. Sudbrack, K.E. Yoon, G. Martin, D.N. Seidman, Nat. Mater. 6, 210 (2006)

16. D. Seidman, C. Sudbrack, K. Yoon, JOM 58 (12), 34 (2006).

of efficient processing and data-mining tools, which involves disciplines that reach beyond the evaporation physics of APT. Solving these mathematical and statistical issues could further broaden the range of addressable materials sciencerelated questions. In this issue, the article by Vurpillot et al. describes and evaluates recent progress in numerical dataprocessing methods.

\section{Summary}

The APT technique today is becoming more and more indispensable for advanced materials characterization. This increasing interest stems from the unique capability of the technique for collecting individual ions from nanoscopic volumes even in complex morphologies, with single-ion sensitivity and mass-resolving power. Recent developments in the instrumentation, in sample preparation, as well as data acquisition and processing enable the applicability of APT to a steadily increasing range of problems. In some extreme cases, APT represents the ultimate characterization method, delivering crucial and decisive information.

\section{Acknowledgments}

Y.A. wishes to acknowledge generous support from the German-Israeli Foundation for Research and Development (GIF), Grant No. I-2333-1150.10/2012 and from the Israel Science Foundation (ISF), Grant No. 698/13. G.S. is grateful to the Deutsche Forschungsgemeinschaft for continuous
17. C.K. Sudbrack, D. Isheim, R.D. Noebe, N.S. Jacobson, D.N. Seidman, Microsc. Microanal. 10 (3), 355 (2004).

18. C.K. Sudbrack, R.D. Noebe, D.N. Seidman, Acta Mater. 55 (1), 119 (2007).

19. C.K. Sudbrack, K.E. Yoon, R.D. Noebe, D.N. Seidman, Acta Mater. 54 (12), 3199 (2006).

20. C.K. Sudbrack, T.D. Ziebell, R.D. Noebe, D.N. Seidman, Acta Mater. 56, 448 (2008).

21. K.E. Yoon, R.D. Noebe, D.N. Seidman, Acta Mater. 55, 1145 (2007).

22. K.E. Yoon, R.D. Noebe, D.N. Seidman, Acta Mater. 55, 1159 (2007).

23. M.D. Mulholland, D.N. Seidman, Acta Mater. 59 (5), 1881 (2011).

24. Y. Amouyal, Z. Mao, D.N. Seidman, Appl. Phys. Lett. 95 (16), 161909 (2009).

25. Y. Amouyal, D.N. Seidman, Acta Mater. 59, 3321 (2011).

26. J. Riley, S. Padalkar, Q. Li, P. Lu, D. Koleske, J. Wierer, G. Wang, L. Lauhon, Nano Lett. 13 (9), 4317 (2013).

27. A. Portavoce, K. Hoummada, I. Berbezier, A. Ronda, D. Mangelinck, Appl. Phys. Lett. 100 (16), 4 (2012).

28. C. Monachon, D.C. Dunand, D.N. Seidman, Small 6 (16), 1728 (2010).

29. M.E. Krug, D.C. Dunand, D.N. Seidman, Appl. Phys. Lett. 92 (12), 124107 (2008).

30. M.E. Krug, D.C. Dunand, D.N. Seidman, Acta Mater. 59 (4), 1700 (2011)

31. Y. Amouyal, Z. Mao, D.N. Seidman, Acta Mater. 58 (18), 5898 (2010).

32. K. Biswas, J. He, I.D. Blum, C.-I. Wu, T.P. Hogan, D.N. Seidman, V.P. Dravid, M.G. Kanatzidis, Nature 489 (7416), 414 (2012).

33. J. He, I.D. Blum, H.-Q. Wang, S.N. Girard, J. Doak, L.-D. Zhao, J.-C. Zheng, G. Casillas, C. Wolverton, M. Jose-Yacaman, D.N. Seidman, M.G. Kanatzidis, V.P. Dravid, Nano Lett. 12 (11), 5979 (2012).

34. J.L. Lensch-Falk, J.D. Sugar, M.A. Hekmaty, D.L. Medlin, J. Alloys Compd. 504 (1), 37 (2010).

35. Y. Pei, J. Lensch-Falk, E.S. Toberer, D.L. Medlin, G.J. Snyder, Adv. Funct. Mater. 21 (2), 241 (2011)

36. B. Gault, E.A. Marquis, D.W. Saxey, G.M. Hughes, D. Mangelinck, E.S. Toberer, G.J. Snyder, Scr. Mater. 63 (7), 784 (2010).

37. I. Blum, D. Isheim, D. Seidman, J. He, J. Androulakis, K. Biswas, V. Dravid, M. Kanatzidis, J. Electron. Mater. 41 (6), 1583 (2012).

38. T.M. Tritt, Annu. Rev. Mater. Res. 41 (1), 433 (2011).

39. G.J. Snyder, E.S. Toberer, Nat. Mater. 7 (2), 105 (2008).

40. D.L. Medlin, G.J. Snyder, Curr. Opin. Colloid Interface Sci. 14 (4), 226 (2009).

41. Y. Amouyal, J. Electron. Mater. 43 (10), 3772 (2014). 
42. T.F. Kelly, D.J. Larson, K. Thompson, R.L. Alvis, J.H. Bunton, J.D. Olson, B.P. Gorman, Annu. Rev. Mater. Res. 37, 681 (2007)

43. K.E. Yoon, D.N. Seidman, C. Antoine, P. Bauer, Appl. Phys. Lett. 93 (13), 132502 (2008).

44. D.E. Perea, J.E. Allen, S.J. May, B.W. Wessels, D.N. Seidman, L.J. Lauhon, Nano Lett. 6 (2), 181 (2006).

45. O. Moutanabbir, D. Isheim, H. Blumtritt, S. Senz, E. Pippel, D.N. Seidman, Nature 496 (7443), 78 (2013).

46. T.J. Prosa, S. Kostrna Keeney, T.F. Kelly, J. Microsc. 237, 155 (2010).

47. Y. Zhang, A.C. Hillier, Anal. Chem. 82, 6139 (2010).

48. B. Gault, W. Yang, K.R. Ratinac, R. Zheng, F. Braet, S. Ringer, Langmuir 26, 5291 (2010).

49. A. Stoffers, C. Oberdorfer, G. Schmitz, Langmuir 28, 56 (2012).
50. T.F. Kelly, 0. Nishikawa, J.A. Panitz, T.J. Prosa, MRS Bull. 34 (10), 744 (2009)

51. L.M. Gordon, L. Tran, D. Joester, ACS Nano 6 (12), 10667 (2012).

52. L.M. Gordon, D. Joester, Nature 469 (7329), 194 (2011).

53. Y.M. Chen, T. Ohkubo, M. Kodzuka, K. Morita, K. Hono, Scr. Mater. 61 (7) 693 (2009).

54. E.A. Marquis, N.A. Yahya, D.J. Larson, M.K. Miller, R.I. Todd, Mater. Today 13 (10), 34 (2010).

55. D.N. Seidman, Rev. Sci. Instrum. 78 (3), 030901/1 (2007).

56. B. Gault, M.P. Moody, J.M. Cairney, S.P. Ringer, Atom Probe Microscopy (Springer Science \& Business Media, 2012).

57. B. Deconihout, P. Gerard, M. Bouet, A. Bostel, Applied Surface Science 94/95, pp. 422-427 (1996)

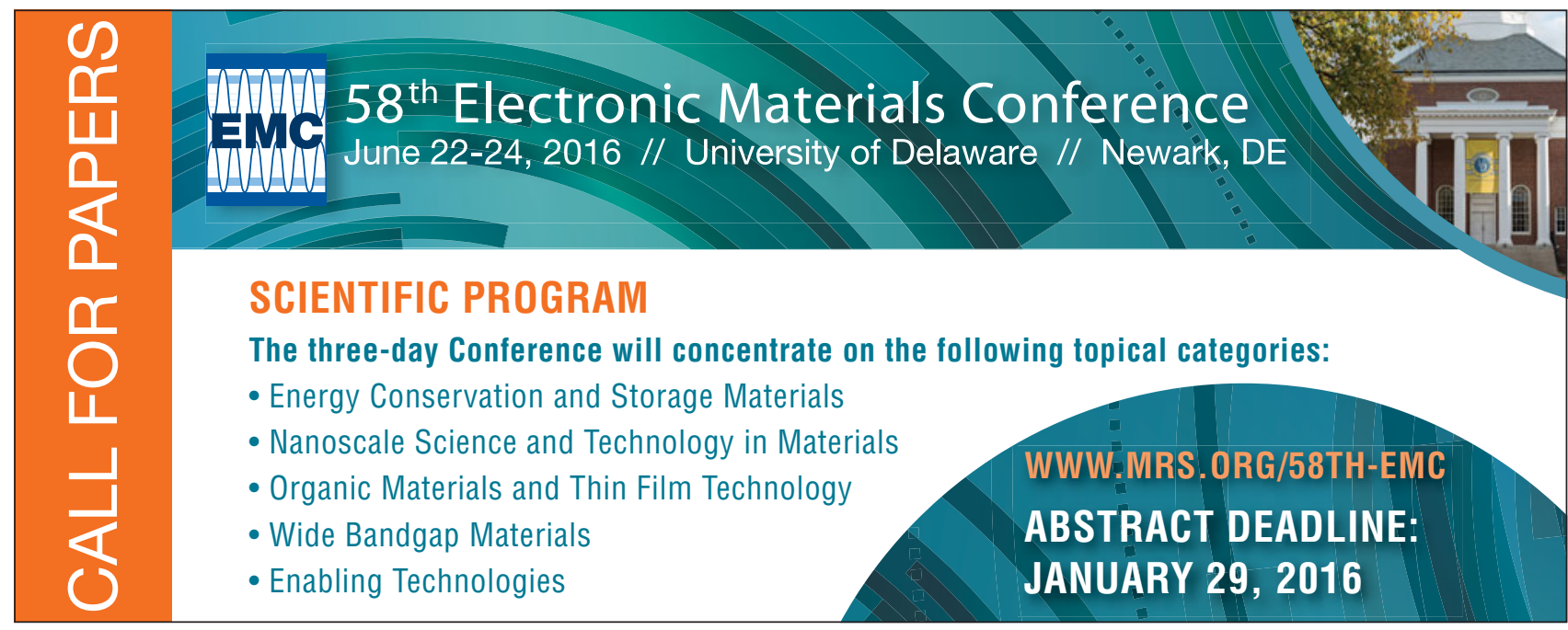

\section{High Resolution}

National Electrostatics Corporation has added Ångstrom level, High Resolution RBS to the RC43 Analysis System for nanotechnology applications. A single Pelletron instrument can now provide RBS, channeling RBS, microRBS, PIXE, ERDA, NRA, and HR-RBS capability, collecting up to four spectra simultaneously. Pelletron accelerators are available with ion beam energies from below $1 \mathrm{MeV}$ in to the $100 \mathrm{MeV}$ region.

www.pelletron.com

Phone: 608-831-7600

E-mail: nec@pelletron.com

\section{National Electrostatics corre.}

